19 THE PREVALENCE OF PRENATAL NEURODEVELOPMENTAL RISK FACTORS IN PREMATURE INFANTS IN CROATIA

Urelija Rodin*, Željka Draušnik, Aida Mujkić, Ivan Cerovečki. Hrvatski zavod za javno zdravstvo

10.1136/archdischild-2021-europaediatrics.19

Objective To determine the prevalence of selected prenatal neurodevelopmental risk factors in premature infants in Croatia.

Methods The medical records used in this study were collected via the National Medical Birth Record Database of the Croatian Institute of Public Health and pertained to all liveborn children delivered before 37 weeks of gestation in 2018 . The data were subsequently extracted from the said records in order to analyse the prevalence of the following prenatal neurodevelopmental risk factors as defined in the International Classification of Diseases, 10th revision: chorioamnionitis (O41.1), placental or umbilical cord disorders (O43 - O45, O69), hypertensive disorders during pregnancy (O10 - O16), gestational diabetes (O24) and smoking during pregnancy. When applicable, causes of early neonatal death among premature infants were analysed as well.

Results 2,232 premature infants were recorded in Croatia in 2018 , i.e. $6 \%$ of all live-born children. 116 (5.2\% of all premature infants) were delivered between 22 and 27 weeks of gestation, $232(10.4 \%)$ between 28 and 31 weeks of gestation and 1,884 (84.4\%) between 32 and 36 weeks of gestation. The selected neurodevelopmental risk factors were recorded in $574(25.7 \%)$ premature infants. The prevalence rates of selected pregnancy-related complications/disorders were as follows: placental or umbilical cord disorders in 169 (7.6\%) premature infants, hypertensive disorders in 160 (7.2\%), smoking during pregnancy in 128 (5.7\%), gestational diabetes in 120 $(5.4 \%)$, chorioamnionitis in 77 (3.5\%). Multiple risk factors were recorded in $74(12.9 \%)$ premature infants at neurodevelopmental risk. Neurodevelopmental risk factors were recorded in 74 infants delivered prior to 27 weeks of gestations (prevalence rate: 637.9/1,000), 95 infants delivered between 28 and 31 weeks $(409.5 / 1,000)$, and 485 infants delivered between 32 and 36 weeks $(257.4 / 1,000)$. The risk of neurodevelopmental disorders was thus 1.6 and 2.5 times higher among infants delivered between 22 and 27 weeks of gestation compared with infants delivered between 28 and 31 weeks of gestation and between 32 and 36 weeks of gestation, respectively. The most frequently recorded neurodevelopmental risk factors by gestational age groups were chorioamnionitis in infants delivered before 27 weeks of gestation (44.6\%), placental or umbilical cord disorders among infants delivered between 28 and 31 weeks of gestation (32.6\%), and hypertensive disorders in infants delivered between 32 and 36 weeks of gestation (27.2\%). 78 early neonatal death were recorded in the studied group (mortality rate: 35.0/1,000); mortality rates by gestational age groups were 431.0/1,000 among infants delivered between 22 and 27 weeks of gestation, 77.6/1,000 among infants delivered between 28 and 31 weeks of gestation and 5.3/1,000 among infants delivered between 32 and 36 weeks of gestation. Chorioamnionitis was the leading cause of death among infants delivered between 22 and 27 weeks of gestation (specific mortality rate: 146.6/1.000); other significant causes of mortality in this age group were prematurity-related disorders (respirational distress disorder, pulmonary atelectasis, pulmonary haemorrhage, intraventricular bleeding (112,1/
1.000). Conversely, congenital malformations were the leading cause of death in infants delivered between 28 and 31 weeks of gestation (30.2/1,000) and infants delivered between 32 and 36 weeks of gestation $(3.7 / 1,000)$.

Conclusion The negative association between the duration of pregnancy and the prevalence of neurodevelopmental risk factors in infants has been confirmed by this study. The prevalence of particular risk factors, however, differed among different gestational age groups. Chorioamnionitis was the most prevalent neurodevelopmental risk factor and most frequent cause of death among infants delivered between 22 and 27 weeks of gestation. Placental and umbilical cord disorders were the most prevalent risk factor among infants delivered between 28 and 31 weeks of gestation, whereas hypertensive disorders most prevalent risk factor among infants delivered between 32 and 36 weeks of gestation. Both groups of disorders are associated with hypoxia and neurodevelopmental disabilities. The Perinatal Health Care Service Programme, recently adopted in Croatia, defines various health care procedures allowing for early diagnosis of pregnancy-related infectious and hypertensive disorders, as well as ultrasound screening for placental circulation disorders. These procedures may contribute to early detection of neurodevelopmental risk factors and prevention of neurodevelopmental disorders.

\section{PREVENTIVE HEALTH CARE OF INFANTS AND PRESCHOOL CHILDREN IN PRIMARY HEALTH CARE PRACTICES IN CROATIA IN 2018}

'Željka Draušnik*, 'Urelija Rodin, ${ }^{1}$ Ivan Cerovečki, ${ }^{2}$ Aida Mujkić. ${ }^{1}$ Croatian Institute of Public Health; ${ }^{2}$ University of Zagreb School of Medicine, Andrija Štampar School of Public Health

\subsection{6/archdischild-2021-europaediatrics.20}

Objective To present prevention activities in primary health care of infants and preschool children in Croatia during 2018, based on data from Central Health Information System of the Republic of Croatia (CEZIH).

Methods Health care of infants (up to one year of age) and preschool children (1-6 years of age) is in Croatia provided within the pre-school children's health service and the family (general) medicine service. More than $90 \%$ of children is in care of primary pediatricians and the rest of them in the care of general practitioners. Practices which are contracted by Croatian Health Insurance Fund routinely send electronic messages with information about the patient and individual visit through CEZIH. As of 2018, Croatian Institute of Public Health also has partial access to those data, for the purpose of analyzing preventive and curative work of primary health care services. In this paper, based on CEZIH data, preventive visits of infants and preschool children in both pre-school children's health service and family (general) medicine service in 2018 are analyzed. The term 'preventive visit' refers to any contact with primary health-care providers: visit, examination, telephone counselling and other contacts for preventive procedures. Each of these procedures has its own code in the list of diagnostic and therapeutic procedures.

Results During 2018, total number of health care users aged 0 to 6 was 272,043, with 2,611,939 visits. Among them, there were 37,619 infants with 533,880 visits, an average of 14.2 visits per infant, while preschool children had an average of 8.9 visits. Among the total visits, 504,469 preventive visits were recorded (19.3\% of all visits), of which 327,637 were 